\title{
Pharyngeal instillation of surfactant before the first breath for prevention of morbidity and mortality in preterm infants at risk of respiratory distress syndrome (Review)
}

Abdel-Latif ME, Osborn DA

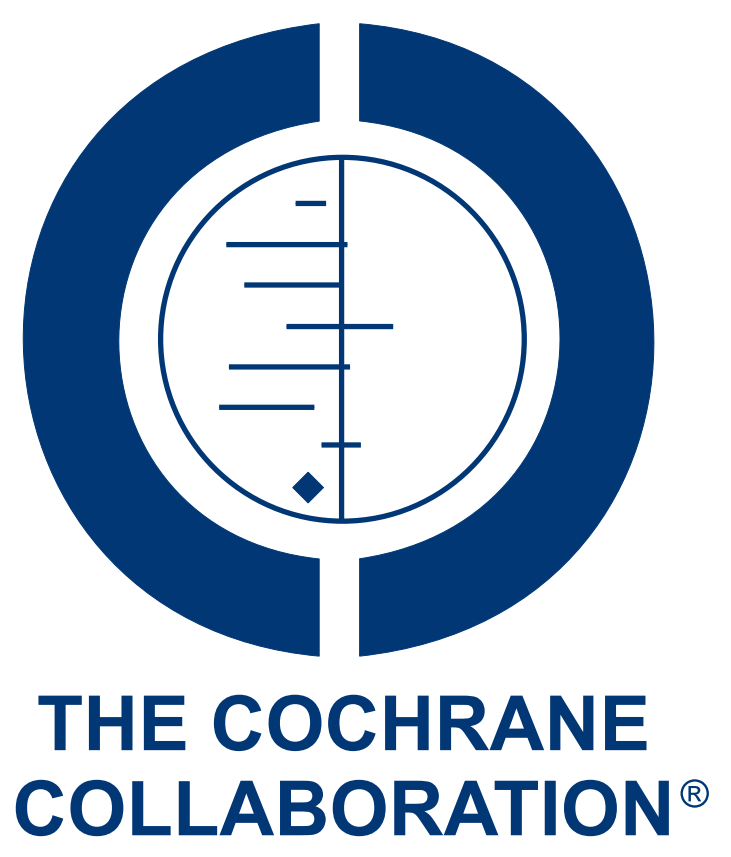

This is a reprint of a Cochrane review, prepared and maintained by The Cochrane Collaboration and published in The Cochrane Library 2011, Issue 3

http://www.thecochranelibrary.com

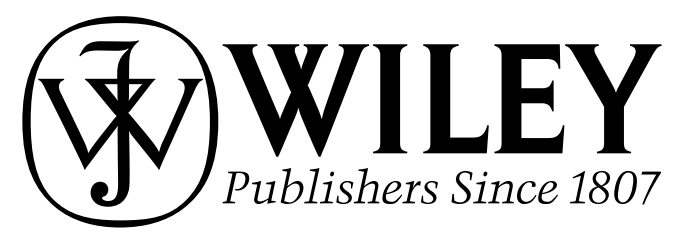

Pharyngeal instillation of surfactant before the first breath for prevention of morbidity and mortality in preterm infants at risk of respiratory distress syndrome (Review)

Copyright (๑) 201 I The Cochrane Collaboration. Published by John Wiley \& Sons, Ltd. 
TABLE OF CONTENTS

HEADER . . . . . . . . . . . . . . . . . . . . . . . . . . . . . . . . . . . . . . . . . 1

ABSTRACT . . . . . . . . . . . . . . . . . . . . . . . . . . . . . . . . . . . . . . 1

PLAIN LANGUAGE SUMMARY . . . . . . . . . . . . . . . . . . . . . . . . . . . . . . . . . . . $\quad . \quad 2$

BACKGROUND . . . . . . . . . . . . . . . . . . . . . . . . . . . . . . . . . . . . . 2

OBJECTIVES . . . . . . . . . . . . . . . . . . . . . . . . . . . . . . . . . . . . . . . . . . . . . . . 4

METHODS . . . . . . . . . . . . . . . . . . . . . . . . . . . . . . . . . . . . . . 4

RESULTS . . . . . . . . . . . . . . . . . . . . . . . . . . . . . . . . . . . . . . . 7

DISCUSSION . . . . . . . . . . . . . . . . . . . . . . . . . . . . . . . . . . . . . 8

AUTHORS' CONCLUSIONS . . . . . . . . . . . . . . . . . . . . . . . . . . . . . . . . . . . . . . . . . 9

ACKNOWLEDGEMENTS . . . . . . . . . . . . . . . . . . . . . . . . . . . . . . . . . . . . . . . . . . . . . 9

REFERENCES . . . . . . . . . . . . . . . . . . . . . . . . . . . . . . . . . . . . . 9

CHARACTERISTICS OF STUDIES . . . . . . . . . . . . . . . . . . . . . . . . . . . . . . . . . . . $\quad$.

DATA AND ANALYSES . . . . . . . . . . . . . . . . . . . . . . . . . . . . . . . . . . . . . . . . . . $\quad .14$

HISTORY . . . . . . . . . . . . . . . . . . . . . . . . . . . . . . . . . . . . . . . 14

CONTRIBUTIONS OF AUTHORS . . . . . . . . . . . . . . . . . . . . . . . . . . . . . . . . . . . . . . . 14

DECLARATIONS OF INTEREST . . . . . . . . . . . . . . . . . . . . . . . . . . . . . . . . . . . 14

DIFFERENCES BETWEEN PROTOCOL AND REVIEW . . . . . . . . . . . . . . . . . . . . . . . . 14

Pharyngeal instillation of surfactant before the first breath for prevention of morbidity and mortality in preterm infants at risk of respiratory distress syndrome (Review)

Copyright @ 201 I The Cochrane Collaboration. Published by John Wiley \& Sons, Ltd. 


\title{
Pharyngeal instillation of surfactant before the first breath for prevention of morbidity and mortality in preterm infants at risk of respiratory distress syndrome
}

\author{
Mohamed E Abdel-Latif ${ }^{1}$, David A Osborn ${ }^{2}$ \\ ${ }^{1}$ Department of Neonatology, Australian National University Medical School, Woden, Australia. ${ }^{2}$ Department of Mothers and Babies \\ NICU, Royal Prince Alfred Hospital, Camperdown, Australia \\ Contact address: Mohamed E Abdel-Latif, Department of Neonatology, Australian National University Medical School, PO Box 11, \\ Woden, ACT, 2606, Australia. Abdel-Latif.Mohamed@act.gov.au.
}

Editorial group: Cochrane Neonatal Group.

Publication status and date: New, published in Issue 3, 2011.

Review content assessed as up-to-date: 16 October 2010.

Citation: Abdel-Latif ME, Osborn DA. Pharyngeal instillation of surfactant before the first breath for prevention of morbidity and mortality in preterm infants at risk of respiratory distress syndrome. Cochrane Database of Systematic Reviews 2011, Issue 3. Art. No.: CD008311. DOI: 10.1002/14651858.CD008311.pub2.

Copyright (C) 2011 The Cochrane Collaboration. Published by John Wiley \& Sons, Ltd.

\begin{abstract}
A B S T R A C T
Background

Intrapartum pharyngeal instillation of surfactant before the first breath may result in surfactant administration to the infant lung, with the potential benefit of avoiding endotracheal intubation and ventilation, ventilator induced lung injury and bronchopulmonary dysplasia.
\end{abstract}

\section{Objectives}

To determine the effect of pharyngeal instillation of surfactant before the first breath compared to placebo, no treatment or intratracheal surfactant administration followed by intermittent positive pressure ventilation (IPPV) on morbidity and mortality in preterm infants at risk of respiratory distress syndrome (RDS).

Search strategy

Searches were made of CENTRAL (The Cochrane Library, to September 2010), MEDLINE and PREMEDLINE (1950 to September 2010), EMBASE (1980 to 2010) and CINAHL (1982 to 2010). This strategy was supplemented by searches of proceedings of scientific meetings, Google Scholar and reference lists of identified studies, as well as contact with expert informants and surfactant manufacturers.

Selection criteria

Published, unpublished and ongoing randomised controlled or quasi-randomised trials (using individual or cluster allocation) of pharyngeal instillation of surfactant before the first breath compared to placebo or no treatment, or intratracheal surfactant instillation followed by IPPV, on morbidity and mortality in preterm infants at risk of RDS.

Data collection and analysis

Two authors independently assessed study eligibility and quality.

Main results

No published, unpublished or ongoing trials that met the inclusion criteria for this review were found.

Pharyngeal instillation of surfactant before the first breath for prevention of morbidity and mortality in preterm infants at risk of 


\section{Authors' conclusions}

There were no data from randomised controlled or quasi-randomised trials that evaluated the effect of intrapartum instillation of pharyngeal surfactant before the first breath. Evidence from animal and observational human studies suggest that pharyngeal instillation of surfactant before the first breath is potentially safe, feasible and may be effective. Well designed trials are needed.

\section{PLAIN LANGUAGE SUMMARY}

\section{Pharyngeal instillation of surfactant before the first breath for prevention of morbidity and mortality in preterm infants at risk of respiratory distress syndrome}

There is no current evidence from clinical trials to guide the use of pharyngeal instillation of surfactant before the first breath in preterm infants at risk of respiratory distress syndrome.

Respiratory distress syndrome is caused by a deficiency of natural lung detergent (surfactant) and occurs mainly in infants born before term (37 weeks' gestation). The usual treatment includes instilling artificial surfactant directly into the newborn infant's airway followed by mechanical ventilation. However, this process can lead to lung injury which may affect the infant's long-term health. A potential alternative strategy is to instil surfactant into the posterior pharynx as soon as the baby's head appears, just before delivery of the shoulders. Thereafter, resuscitation measures are instituted as usual. This procedure has the potential to reduce the need to support the infant's breathing after birth, as well as any lung damage caused by mechanical ventilation. We did not find any trials for this review of pharyngeal instillation of surfactant before the first breath in preterm infants at risk of respiratory distress syndrome. In view of the encouraging results from animal studies and preliminary human studies, trials of pharyngeal instillation of surfactant before the first breath in preterm infants at risk of respiratory distress syndrome are needed.

\section{B A C K G R O U N D}

\section{Description of the condition}

Respiratory distress syndrome (RDS) results from a deficiency of pulmonary surfactant and is a significant cause of morbidity and mortality in preterm infants. Randomised controlled trials and meta-analyses have demonstrated the efficacy of surfactant therapy in both the prevention and treatment of infants with, or at risk of, RDS. Studies have compared a wide variety of surfactant preparations. These include synthetic surfactants (Soll 1998a; Soll $1998 \mathrm{~b}$ ) and surfactants derived from animal sources (natural surfactants) (Seger 2009; Soll 2002). Although both synthetic and animal-derived surfactant preparations are effective (Soll 1998a; Soll 1998b; Soll 2002; Seger 2009), clinical trials suggest that animalderived surfactant may be more effective (Soll 2001a) than protein-free synthetic surfactant (Tooley 1987). Furthermore, clinical trials have shown prophylactic surfactant (Soll 1998b; Soll 2001b; Soll 2002) and early surfactant (Soll 1999; Stevens 2007) are superior to selective use of surfactant in preventing morbidity and mortality in preterm infants. The trials also suggest that a multi- ple-dose strategy is superior to a single-dose strategy (Soll 2009). Tests also point to the successful use of new protein-containing synthetic surfactants (Pfister 2007) although these preparations are not currently available for clinical use.

Despite the benefits of surfactant, many infants develop bronchopulmonary dysplasia (BPD) and chronic lung disease (CLD). Although the aetiology of CLD in preterm infants is multifactorial (Allen 2003), ventilator-induced lung injury (VILI) remains one of the main implicated risk factors (Coalson 1999; Clark 2000). We know that only a few resuscitative positive pressure ventilation (PPV) breaths may result in VILI (Grossmann 1986; Björklund 1997; Fleckonoe 2008; O’Reilly 2008).

Both surfactant prophylaxis and therapy necessitate endotracheal intubation to facilitate surfactant administration. Although surfactant by itself is an established, effective intervention for either the prevention or treatment of RDS (Soll 1998a; Soll 1998b; Soll 1999; Soll 2001a; Soll 2001b; Soll 2002; Stevens 2007; Seger 2009; Soll 2009), endotracheal intubation and the subsequent use of PPV are not without side effects.

Endotracheal intubation is a potentially traumatic procedure that is often performed without optimal pain management (Sarkar 
2006). It may be accompanied by significant haemodynamic instability including hypoxia, bradycardia, blood pressure fluctuation and an intracranial pressure increase (Marshall 1984; Ghanta 2007). Intubation is inevitably associated with colonisation of the trachea, retained secretions resulting in collapse, differential aeration and high resistance to air flow, resulting in increased work of breathing and potentially leading to nosocomial pneumonia and sepsis (Young 2005; Aly 2008). Intubation is associated with an inflammatory process which can lead to lung injury and BPD (Young 2005). Current evidence suggests PPV of immature, surfactant-deficient lungs is harmful and may exacerbate the development of BPD (Björklund 1997; Van Marter 2000). Björklund reported that resuscitation of surfactant-deficient immature lambs with as few as six breaths damages the lungs and blunts the therapeutic effect of subsequent surfactant replacement (Björklund 1997). Grossmann 1986 reported similar results. Fleckonoe 2008 reported that just six hours of ventilation were enough to cause marked airway epithelial injury in very preterm and near-term fetal sheep. O'Reilly 2008 reported that ventilator-induced injury extends to involving the conducting airways as well.

One approach to surfactant administration is the InSurE (INtubation-SURfactant-Extubation) technique pioneered by Victorin (Victorin 1990) and Verder (Verder 1994). A review of trials found that early surfactant replacement therapy with prompt extubation to nasal continuous positive airway pressure (nCPAP), as in the InSurE technique, is associated with less need for mechanical ventilation, lower incidence of BPD and fewer air leak syndromes when compared with later, selective surfactant replacement and continued mechanical ventilation with extubation from low ventilator support (Stevens 2007). However, the limited data available show the InSurE procedure to be associated with a propensity for decreased cerebral oxygenation, higher cerebral oxygen extraction and decreased electric brain activity (Hellström-Westas 1992; van de Berg 2000). Furthermore, the InSurE procedure may need to be repeated if the first dose of surfactant was not sufficiently effective (Bohlin 2007), leading to additional risk of brain damage. The main strategy used to avoid endotracheal intubation and PPV in premature infants is application of nCPAP or continuous distending pressure (CDP) immediately following birth (Kamper 1999; Ho 2002a; Ho 2002b). Recent studies suggest CDP may lead to less CLD compared to elective intubation, surfactant and PPV (Aly 2001; de Klerk 2001). Similarly, nasal intermittent positive pressure ventilation (NIPPV) has shown a decreased need for intubation and mechanical ventilation. It also reduces the frequency of apnoea and the incidence of CLD without an increase in adverse effects (Davis 2001; Lemyre 2002). Although CDP and NIPPV strategies avoid endotracheal intubation and PPV they preclude surfactant administration, which is a standard and proven treatment for RDS. Furthermore, CDP, NIPPV and InSurE may fail in $25 \%$ to $50 \%$ of preterm infants (Reininger 2005; Kugelman 2007; Morley 2008).

\section{Description of the intervention}

Non-invasive methods of surfactant administration have the potential to reduce the need for intubation and endotracheal surfactant administration. Potential strategies include:

1. intra-amniotic instillation (Petrikovsky 1995);

2. pharyngeal instillation (Kattwinkel 2002; Kattwinkel 2004);

3. administration of airway surfactant via a laryngeal mask (Trevisanuto 2005);

4. administration via a thin endotracheal catheter without IPPV (Kribs 2007; Dargaville 2010; Kribs 2010);

5. nebulised surfactant administration in spontaneously breathing infants (Jorch 1997).

This review focuses on pharyngeal instillation of surfactant. The typical protocol for pharyngeal instillation of surfactant is as follows (Kattwinkel 2002; Kattwinkel 2004): as soon as the baby's head appears on the perineum or at the operative incision, the mother is instructed to stop pushing and the pharynx and stomach are suctioned with a catheter. A surfactant solution is then instilled into the posterior pharynx through a catheter without direct laryngoscopy. The baby is stimulated to breathe spontaneously as soon as the shoulders and rest of the body are delivered. Thereafter, resuscitation measures are instituted as usual.

\section{How the intervention might work}

Pharyngeal instillation of surfactant is designed to administer surfactant to the surfactant-deficient lungs by taking advantage of the normal physiology of establishing breathing air. While the chest remains compressed in the birth canal, fetal lung fluid is suctioned from the future upper airway and is replaced with a surfactant-containing solution. The chest expands as it is released into the vaginal vault and beyond and the baby is stimulated to aspirate the surfactant-containing solution, providing surfactant at the advancing air-fluid interface. The technique is designed to avoid endotracheal intubation whilst offering the benefits of surfactant administration. Combining pharyngeal surfactant administration with prenatal steroid administration and CDP offers potential synergy to treat RDS, avoiding both endotracheal intubation and PPV, thereby reducing lung injury that may lead to BPD.

Animal studies in rabbits and lambs indicate that surfactant deposition into the posterior pharynx before the first breath permits aspiration of surfactant at initiation of ventilation, improves expansion of the lungs and prolongs survival (Enhorning 1973; Cummings 1995). Furthermore, animal studies have shown that surfactant is more uniformly distributed (Jobe 1984) and pulmonary function maintained for a longer time (Cummings 1995) if surfactant is delivered to fluid-filled rather than air-filled lungs.

\section{Why it is important to do this review}


Despite significant advances in neonatal intensive care, CLD results in a significant health burden to preterm infants born at less than 32 weeks' gestation who received mechanical ventilation. CLD results in substantial neonatal and infant morbidities and health resource utilisation (Allen 2003). It is associated with chronic respiratory difficulties (Kilbride 2003; Doyle 2006); prolonged and recurrent hospitalisation (Chye 1995); neurodevelopmental disabilities including cerebral palsy, neurosensory and motor disabilities (Skidmore 1990; Singer 1997; Majnemer 2000) and poor cognitive outcome (Hughes 1999). CLD has a major impact on the daily lives of families, which persists beyond the neonatal period (Korhonen 1999). Pharyngeal instillation of surfactant before the first breath is a physiologic and logical technique with the potential benefit of avoiding ventilation, VILI and possibly BPD.

\section{O B J E C T I VES}

To determine the effect of pharyngeal instillation of surfactant before the first breath compared to placebo, no treatment or intratracheal surfactant administration followed by intermittent positive pressure ventilation (IPPV) on morbidity and mortality in preterm infants at risk of RDS.

\section{METHODS}

\section{Criteria for considering studies for this review}

\section{Types of studies}

Trials using randomisation or quasi-randomisation of patients regardless of the unit of allocation (individual or cluster) were eligible for inclusion. Published or unpublished and ongoing studies were eligible for inclusion.

\section{Types of participants}

Participants were mother-infant dyads. We included preterm infants of less than 32 completed weeks' gestation that were at risk of RDS. Infants should have had the intervention attempted before the first breath to be eligible.

\section{Types of interventions}

Pharyngeal instillation of surfactant, attempted before the first breath at any dose and using any type of surfactant (synthetic, animal-derived or protein-containing synthetic), compared with placebo, no treatment or intratracheal instilled surfactant. We planned to perform separate comparisons for all the above groups.

\section{Types of outcome measures}

\section{Primary outcomes}

Primary outcome measures included:

1. chronic lung disease (CLD), defined as need for oxygen or respiratory support at 36 weeks postmenstrual age (PMA);

2. mortality prior to hospital discharge;

3. neurodevelopmental disability at a minimum of 18 months postnatal age, defined as neurological abnormality including cerebral palsy on clinical examination, developmental delay of more than two standard deviations below the population mean on a standardized test of development, blindness (visual acuity less than 6/60) or deafness (any hearing impairment requiring amplification) at any time after the age was term corrected.

\section{Secondary outcomes}

Secondary outcome measures included other measures of effectiveness, complications, satisfaction with care and health service use for both women and infants. These included any of the following.

\section{A. Infants}

1. Fetal trauma during intervention

2. Bradycardia during intervention

3. Discontinuation of intervention because of fetal side effects (e.g. bradycardia)

4. Apgar score less than 7 at five minutes

5. Metabolic acidaemia ( $\mathrm{pH}$ less than 7.05 or base deficit greater than $12 \mathrm{mmol} / \mathrm{L}$, or both) in cord artery blood

6. Perinatal asphyxia (Stage 1 to 3: Sarnat and Sarnat Stages of Hypoxic-Ischaemic Encephalopathy)

7. Need for positive pressure ventilation via neopuff or bag and mask during resuscitation at delivery

8. Need for intubation during resuscitation at delivery

9. Need for chest compression during resuscitation at delivery

10. Need for adrenaline during resuscitation at delivery

11. Intratracheal surfactant received post-intervention

12. Doses of post-intervention surfactant

13. Hypothermia post resuscitation (a core body temperature of less than $36.5^{\circ} \mathrm{C}$ or a skin temperature of less than $36^{\circ} \mathrm{C}$ )

14. Respiratory distress syndrome (however defined by authors)

15. Need for mechanical ventilation

16. Days of mechanical ventilation

17. Days of continuous positive airway pressure (CPAP)

18. Days of high-flow nasal cannula

19. Days of low-flow nasal cannula

20. Use of high-frequency oscillatory ventilation (HFOV) as a rescue treatment for respiratory distress 
21. Use of jet ventilation as a rescue treatment for respiratory distress

22. Use of extracorporeal membrane oxygenation (ECMO) as a rescue treatment for respiratory distress

23. Use of postnatal corticosteroids as rescue treatment for respiratory distress

24. Days of supplemental oxygen

25. Incidence of pulmonary interstitial emphysema

26. Incidence of pneumothorax

27. Chronic lung disease (CLD) defined as need for oxygen at 28 days of age

28. Use of diuretic as prophylaxis or rescue treatment for CLD

29. Use of postnatal corticosteroid as prophylaxis or rescue treatment for CLD

30. Use of home oxygen

31. Apnoea treated with methylxanthines or CPAP

32. Hypotension (however defined by authors) requiring inotropic support

33. Systemic infection - early (first 48 hours of life) and late

34. Intraventricular haemorrhage (any and severe: Papile grade equal to or greater than 3 )

35. Periventricular leukomalacia (however defined by authors)

36. Symptomatic patent ductus arteriosus, or treated with cyclo-oxygenase inhibitors or surgical ligation

37. Proven necrotising enterocolitis (Bell stage equal to or greater than 2)

38. Retinopathy of prematurity (any and severe: stage equal to or greater than 3 )

39. Neonatal mortality (less than 28 days of age)

40. Days to regain birthweight

41. Postnatal growth failure (e.g. weight at discharge less than 10th percentile)

42. Duration of hospitalisation (days)

43. Asthma diagnosed by physician or challenge test

44. Rehospitalization for asthma

45. Rehospitalization for hyperactive airway disease

46. Rehospitalization for pneumonia

B. Mother

1. Discontinuation of intervention because of maternal side effect or discomfort

2. Maternal satisfaction or stress as measured on validated scale

3. Postpartum haemorrhage (clinically estimated blood loss greater than or equal to $500 \mathrm{ml}$ at time of birth and up to 24 hours)

4. Interval from pharyngeal surfactant instillation to delivery

5. Length of maternal hospitalisation (days)

\section{Search methods for identification of studies}

See: Cochrane Handbook for Systematic Reviews of Interventions Handbook (Cochrane Handbook 2008) for methods used in reviews.
We used the standard search strategy of the Cochrane Neonatal Review Group as outlined in The Cochrane Library. We considered unpublished studies as eligible for review. The search of MEDLINE and PREMEDLINE (via the Ovid interface) included the following $\mathrm{MeSH}$ terms and text words: "infant, premature, preterm, newborn, neonate", "surfactant", "laryngeal“, "mask", "airway". We limited our searches to "randomized and quasi-randomised clinical trials”. We did not apply language restrictions. The search strategy for MEDLINE and PREMEDLINE was as follows.

\#1 exp pregnancy

\#2 exp infant premature

\#3 exp infant newborn

\#4 exp obstetric labor premature

\#5 exp premature birth

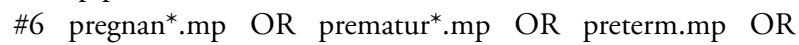
neonat*.mp OR infant*.mp OR newborn.mp

\#7 \#1 OR \#2 OR \#3 OR \#4 OR \#5 OR \#6

\#8 pharyngeal.mp

\#9 oral.mp OR mouth

\#10 nasl.mp

\#11 oropharynx.mp

\#12 nasopharynx.mp

\#13 intrapartum.mp

$\# 14$ intra-partum.mp

\#15 \#8 OR \#9 OR \#10 OR \#11 OR \#12 OR \#13 OR \#14

\#16 exp pulmonary surfactants

\#17 surfactant*.mp OR Beractant.mp OR Poractant.mp OR Curosurf.mp OR Survanta.mp OR Exosurf.mp OR Lucinactant.mp

\#18 \#15 OR \#16 OR \#17

\#19 \#7 AND \#15 AND \#18

We adapted the above search strategy to suit other electronic sources.

\section{Electronic searches}

We adapted the search strategy above to search the following electronic databases:

1. The Oxford Database of Perinatal Trials;

2. Cochrane Central Register of Controlled Trials (CENTRAL); 3. MEDLINE and PREMEDLINE (1966 to 2010) via OVID interface;

4. EMBASE (1980 to 2010) via OVID interface;

5. CINAHL (1982 to 2010) via EBSCO interface;

6. Google Scholar.

\section{Searching other resources}

We carried out additional searches as follows.

1. Ongoing trials in the following trial registries (searched October 2010):

Pharyngeal instillation of surfactant before the first breath for prevention of morbidity and mortality in preterm infants at risk of 
- ClinicalTrials.gov (U.S. National Institutes of Health),

- Current Controlled Trials,

- Australian New Zealand Clinical Trials Registry,

- International Clinical Trials Registry Platform (ICTRP).

2. Abstracts of conferences from:

- Proceedings of Pediatric Academic Societies (American Pediatric Society, Society for Pediatric Research and European Society for Pediatric Research) (from 1990 to 2010) from the journal "Pediatric Research" and abstracts online;

- Proceedings of European Academy of Paediatric Societies (EAPS), The European Society for Paediatric Research (ESPR), European Academy of Paediatrics (EAP) and European Society of Paediatric and Neonatal Intensive Care (ESPNIC) (2003 to 2009) from abstracts online;

- Proceedings of Perinatal Society of Australia and New Zealand (PSANZ) (1996 to 2010) handsearched from the published proceedings.

3. Reference lists. After reading the identified individual studies that examined the effect of pharyngeal surfactant installation on morbidity and mortality in preterm infants at risk of RDS, we screened the reference lists of these papers to identify other relevant studies

4. Personal communications with expert informants and authors of included studies.

5. Pharmaceutical companies. We contacted the company (Abbott Laboratories, Inc) that developed different types of surfactant for possible unpublished studies using their products.

\section{Data collection and analysis}

We planned to use the standardized review method of the Cochrane Neonatal Review Group (CNRG) for conducting a systematic review (http://neonatal.cochrane.org/en/index.html). We planned to enter and cross-check data using Review Manager 5 software (RevMan 2008).

\section{Selection of studies}

Both review authors independently assessed study eligibility for inclusion in this review. We retrieved full text versions for potentially eligible studies when the abstract provided inadequate information.

\section{Data extraction and management}

Both review authors independently extracted data from the fulltext articles using specifically designed spreadsheets to manage the information. We planned to use these forms to decide on trial inclusion and exclusion, extract data from eligible trials and to request additional published information from authors of the original reports. We planned to compare the extracted data for any differences. If noted, we planned to resolve differences through mutual discussion and consensus.

\section{Assessment of risk of bias in included studies}

We planned to use the standardized review methods of The Cochrane Collaboration to assess the methodological quality of studies (Higgins 2008). The review authors planned to independently assess the quality and risk of bias of the potential studies based on the following six domains:

1) sequence generation;

2) allocation concealment;

3) blinding of participants, personnel and outcome assessors;

4) incomplete outcome data;

5) selective outcome reporting;

6) other sources of bias.

When necessary, we planned to request additional information and clarification of published data from the authors of individual trials. We planned to assess each trial for risk of bias based on the criteria listed above and mark as:

a) low risk of bias;

b) unclear risk of bias;

c) high risk of bias.

We planned to resolve discrepancies by consensus and to report any persisting discrepancies.

\section{Measures of treatment effect}

We planned to report dichotomous data using relative risk (RR) and risk difference (RD) with $95 \%$ confidence intervals (CI). If there was a statistically significant reduction in $\mathrm{RD}$ we planned to calculate the number needed to treat (NNT) and associated 95\% CI. We planned to report mean differences with $95 \%$ CI for continuous outcomes.

\section{Unit of analysis issues}

The unit of randomisation is the intended unit of analysis and is expected to be individual infants.

\section{Cluster-randomised trials}

We planned to include cluster-randomised trials in the analyses along with individually randomised trials. We planned to analyse them using the methods described in the Cochrane Handbook for Systematic Reviews of Interventions (Higgins 2008) using an estimate of the intra-cluster correlation coefficient (ICC) derived from the trial (if possible) or from another source. If we use ICCs from other sources, we planned to report this and conduct sensitivity analyses to investigate the effect of variation in the ICC. If we identify both cluster-randomised trials and individually-randomised trials, we planned to synthesise the relevant information. We considered it reasonable to combine the results from both if 
there is little heterogeneity between the study designs and interaction between the effect of intervention and the choice of randomisation unit is unlikely.

\section{Dealing with missing data}

We planned to obtain missing data from the authors, when possible. If this is not possible, we planned to conduct analyses on available data (that is ignoring the missing data). In addition, we planned to conduct another analysis by using imputation methods (both best and worst scenarios) and last observation carried forward (LOCF) to the final assessment method for dichotomous and continuous outcome data, respectively.

For dichotomous outcomes we planned to conduct both best and worst scenarios and intention-to-treat (ITT) analysis with imputation. We planned to compare results obtained from two analysis options to arrive at a better understanding of the robustness of results relative to the different analytic approaches. We planned to consider an imputation approach of best case scenarios (that is all missing participants in the intervention group did not experience a bad outcome such as death or BPD and all missing participants in the control group experienced a bad outcome) and worst case scenarios (that is all missing participants in the intervention group experienced the event and all missing participants in the control condition did not). We planned to conduct a sensitivity analysis to compare results based on different imputation assumptions (that is best versus worst scenarios).

We planned to analyse missing continuous data on an endpoint basis, including only participants with a final assessment or using LOCF to the final assessment if the trial authors reported LOCF data.

\section{Assessment of heterogeneity}

We planned to use Review Manager software version 5 (RevMan 2008) provided by The Cochrane Collaboration to assess the heterogeneity of treatment effects between trials. We planned to establish the two formal statistics described below.

1) The $\mathrm{Chi}^{2}$ test to assess whether observed variability in effect sizes between studies was greater than would be expected by chance. Since this test has low power when the number of studies included in the meta-analysis is small, we will set the probability at the $10 \%$ level of significance.

2) The $I^{2}$ statistic to ensure that pooling of data is valid. We planned to grade the degree of heterogeneity as: $0 \%$ to $30 \%$, might not be important; $31 \%$ to $50 \%$, moderate heterogeneity; $51 \%$ to $75 \%$, substantial heterogeneity; $76 \%$ to $100 \%$, considerable heterogeneity.

Where there is evidence of apparent or statistical heterogeneity, we planned to assess the source of the heterogeneity using sensitivity and subgroup analysis looking for evidence of bias or methodological differences between trials.

\section{Assessment of reporting biases}

We planned to assess reporting and publication bias by examining the degree of asymmetry of a funnel plot in RevMan 5 (RevMan 2008).

\section{Data synthesis}

We planned to perform statistical analyses according to the recommendations of the Cochrane Neonatal Review Group (CNRG) (http://neonatal.cochrane.org/en/index.html). We planned to analyse all infants randomised on an intention-to-treat basis. We planned to analyse treatment effects in the individual trials. In the first instance we planned to use a fixed-effect model to combine the data. For any meta-analyses of categorical outcomes we planned to calculate typical estimates of RR and RD, each with its $95 \%$ CI. For continuous outcomes we planned to calculate the mean difference (MD) if outcomes were measured in the same way between trials. We planned to establish the standardized mean difference (SMD) to combine trials that measured the same outcome but used different scales. Where we consider meta-analysis to be inappropriate, we planned to analyse and interpret individual trials separately.

\section{Subgroup analysis and investigation of heterogeneity}

If sufficient data were available, we planned to explore potential sources of clinical heterogeneity through the following a priori subgroup analyses:

1. dose of surfactant (same dose as intratracheal, more than intratracheal dose);

2. gestational age at delivery (less than 27,27 to 28,29 to 32 completed weeks' gestation);

3. type of surfactant used (synthetic, animal-derived or protein-containing synthetic).

\section{Sensitivity analysis}

If sufficient data were available, we planned to explore methodological heterogeneity through the use of sensitivity analyses. We planned to perform sensitivity analyses by excluding trials of lower quality, based on a lack of any of the following: allocation concealment, adequate randomisation, blinding of treatment, less than $10 \%$ loss to follow up.

\section{RE S U L T S}

\section{Description of studies}

See: Characteristics of excluded studies. 


\section{Results of the search}

The search of the sources above did not find any published, unpublished or ongoing randomised or quasi-randomised controlled trials to be considered for inclusion in this review.

\section{Excluded studies}

One non-randomised study (case series) of pharyngeal instillation of surfactant (Kattwinkel 2002; Kattwinkel 2004) was excluded.

\section{Risk of bias in included studies}

Not applicable, as no eligible studies were found meeting the inclusion criteria for this review.

\section{Effects of interventions}

There are no trials that meet the inclusion criteria for this review.

\section{DISCUSSION}

Pharyngeal instillation of surfactant is designed to administer surfactant to the surfactant-deficient lung by taking advantage of the normal physiology of establishing air breathing. This technique is potentially useful for administration of prophylactic surfactant. In a feasibility study, Kattwinkel 2004 studied a non-random purposive sample of 23 preterm newborns. Generally, there was sufficient time (usually approximately 30 to 45 seconds) for the procedure during vaginal births with vertex presentations, but not vaginal breech or caesarean section. The study showed that nasopharyngeal surfactant instillation at birth appears to be relatively safe and simple to accomplish, especially for vaginal births. No major complications from the study procedure were encountered. However, a large randomised clinical trial (RCT) will be required to determine the efficacy of this technique. Fernandes 2005 discussed certain issues that need to be addressed before applying this technique in randomised trials and clinical practice.

1. Dose of surfactant: it was suggested to use a greater volume of surfactant for nasopharyngeal administration than is used for intratracheal instillation as it is unlikely that the entire dose of surfactant will be aspirated. Alternatively, a more concentrated surfactant solution for nasopharyngeal instillation could be used.

2. Quantification of the amount of surfactant aspirated: this will need to be addressed before embarking on a large RCT. Fernandes 2005 suggested using perflurocarbon-surfactant mixture, which would have the dual advantages of allowing visualization of the radio-opaque perflurocarbon on chest radiograph as well as attenuation of a pulmonary inflammatory response (von der Hardt
2002; von der Hardt 2004), which might contribute to decreased incidence of chronic lung disease.

3. Whether extremely preterm infants (especially $<28$ weeks gestation) are able to muster sufficient respiratory effort to aspirate nasopharyngeal surfactant needs further evaluation.

Animal studies in rabbits and lambs indicate that surfactant deposition into the posterior pharynx before the first breath permits aspiration of surfactant at initiation of ventilation, improves expansion of the lungs and prolongs survival (Enhorning 1973; Cummings 1995). Furthermore, animal studies have shown that surfactant is more uniformly distributed (Jobe 1984) and pulmonary function maintained for a longer time (Cummings 1995) if surfactant is delivered to a fluid-filled rather than air-filled lung.

\section{Summary of main results}

No eligible trials were identified that assessed the effect of pharyngeal instillation of surfactant before the first breath, compared to placebo, no treatment or intratracheal surfactant instillation with IPPV, on morbidity and mortality in preterm infants at risk of RDS.

\section{Overall completeness and applicability of evidence}

Since no eligible trials were identified, there is no evidence from randomised clinical trials to assess the effectiveness of pharyngeal surfactant administration in preterm infants.

\section{Quality of the evidence}

There are no trials that met the inclusion criteria for this review.

\section{Potential biases in the review process}

Searches were made for published and unpublished trials including proceeding from scientific meetings, citations of studies and reviews, expert informants, trial registries and surfactant manufacturers. No trials were found that met the inclusion criteria for this review.

\section{Agreements and disagreements with other studies or reviews}

There are no trials that met the inclusion criteria for this review. 


\section{A U THORS' CONCLUSIONS}

\section{Implications for practice}

There is no evidence from randomised clinical trials to support or refute the use of intrapartum instillation of pharyngeal surfactant. The use of pharyngeal instillation of surfactant should be limited to randomised controlled trials.

\section{Implications for research}

The data available from uncontrolled human and animal studies have shown a potential benefit of intrapartum pharyngeal instillation of surfactant and justify the conduct of randomised controlled trials. Infants enrolled in such trials should be stratified by gestation.

\section{ACKNOWLEDGEMENTS}

As part of the prepublication editorial process, this protocol has been commented on by three peers (an editor and two referees who are external to the editorial team) and the Group's Statistical Adviser.

Editorial support of the Cochrane Neonatal Review Group has been funded with Federal funds from the Eunice Kennedy Shriver National Institute of Child Health and Human Development National Institutes of Health, Department of Health and Human Services, USA, under Contract No. HHSN267200603418C.

\section{REFERENCES}

\section{References to studies excluded from this review}

Kattwinkel 2002 \{published data only\}

Kattwinkel J, Ferguson JE, Bloom BT. Intrapartum pharyngeal surfactant for RDS prophylaxis [Abstract]. Proceedings of the Pediatric Academic Societies (PAS) Annual Meeting, Baltimore, Maryland, USA. 4-7 May 2002:PAS2002:2021.

Kattwinkel 2004 \{published data only\}

Kattwinkel J, Robinson M, Bloom BT, Delmore P, Ferguson JE. Technique for intrapartum administration of surfactant without requirement for an endotracheal tube. Journal of Perinatology 2004; 24(6):360-5.

\section{Additional references}

Allen 2003

Allen J, Zwerlding R, Ehrenkranz R, Gaultier C, Geggel R, Greenough A, et al.Statement on the care of the child with chronic lung disease of infancy and childhood. American Journal of Respiratory and Critical Care Medicine 2003;168(3):356-96.

Aly 2001

Aly HZ. Nasal prongs continuous positive airway pressure: a simple yet powerful tool. Pediatrics 2001;108(3):759-61.

Aly 2008

Aly HZ, Badawy M, El-Kholy A, Nabil R, Mohamed A. Randomized, controlled trial on tracheal colonization of ventilated infants: can gravity prevent ventilator-associated pneumonia?. Pediatrics 2008;122(4):770-4.

Björklund 1997

Björklund LJ, Ingimarsson J, Curstedt T, John J, Robertson B, Werner O, et al.Manual ventilation with a few large breaths at birth compromises the therapeutic effect of subsequent surfactant replacement in immature lambs. Pediatric Research 1997;42(3): 348-55.

Bohlin 2007

Bohlin K, Gudmundsdottir T, Katz-Salamon M, Jonsson B, Blennow M. Implementation of surfactant treatment during continuous positive airway pressure. Journal of Perinatology 2007; 27(7):422-7.

Chye 1995

Chye JK, Gray PH. Rehospitalization and growth of infants with bronchopulmonary dysplasia: a matched control study. Journal of Paediatrics and Child Health 1995;31(2):105-11.

Clark 2000

Clark RH, Slutsky AS, Gerstmann DR. Lung protective strategies of ventilation in the neonate: what are they?. Pediatrics 2000;105(1 Pt 1):112-4.

Coalson 1999

Coalson JJ, Winter VT, Siler-Khodr T, Yoder BA. Neonatal chronic lung disease in extremely immature baboons. American Journal of Respiratory and Critical Care Medicine 1999;160(4):1333-6.

\section{Cummings 1995}

Cummings JJ, Holm BA, Nickerson PA, Ferguson WH, Egan EA. Pre- versus post-ventilatory surfactant treatment in surfactantdeficient preterm lambs. Reproduction, Fertility, and Development 1995;7(5):1333-8.

Dargaville 2010

Dargaville PA, Aiyappan A, Williams C, Cornelius A, de Paoli AG. Preliminary study of a new method of minimally invasive surfactant therapy [Abstract]. Proceedings of the 14th Annual Congress of the Perinatal Society of Australia and New Zealand (PSANZ). Wellington, New Zealand, 28-31 March 2010:A153.

Davis 2001

Davis PG, Lemyre B, De Paoli AG. Nasal intermittent positive pressure ventilation (NIPPV) versus nasal continuous positive airway pressure (NCPAP) for preterm neonates after extubation. Cochrane Database of Systematic Reviews 2001, Issue 3. [DOI: 10.1002/14651858.CD003212]

de Klerk 2001

de Klerk AM, de Klerk RK. Use of continuous positive airway pressure in preterm infants: comments and experience from New Zealand. Pediatrics 2001;108(3):761-2. 


\section{Doyle 2006}

Doyle LW, Victorian Infant Collaborative Study Group.

Respiratory function at age 8-9 years in extremely low birthweight/ very preterm children born in Victoria in 1991-1992. Pediatric Pulmonology 2006;41(6):570-6.

\section{Enhorning 1973}

Enhorning G, Grossman G, Robertson B. Pharyngeal deposition of surfactant in the premature rabbit fetus. Biology of the Neonate 1973;22(1):126-32.

\section{Fernandes 2005}

Fernandes CJ, Horst DA, O’Donovan DJ. Nasopharyngeal surfactant administration to prevent neonatal respiratory distress syndrome [Letter]. Journal of Perinatology 2005;25:361-2.

\section{Fleckonoe 2008}

Fleckonoe SJ, Crossley KJ, Lane MA, Allison BJ, Morley CJ, Hooper SB. The effects of mechanical ventilation on airway epithelium in near term and very preterm sheep [Abstract]. Journal of Paediatrics and Child Health 2008;44:A42.

\section{Ghanta 2007}

Ghanta S, Abdel-Latif ME, Lui K, Ravindranathan H, Awad A, Oei J. Propofol compared with the morphine, atropine, and suxamethonium regimen as induction agents for neonatal endotracheal intubation: a randomized, controlled trial. Pediatrics 2007;119(6):e1248-55.

\section{Grossmann 1986}

Grossmann G, Nilsson R, Robertson B. Scanning electron microscopy of epithelial lesions induced by artificial ventilation of the immature neonatal lung; the prophylactic effect of surfactant replacement. European Journal of Pediatrics 1986;145(5):361-7.

\section{Hellström-Westas 1992}

Hellström-Westas L, Bell AH, Skov L, Greisen G, Svenningsen NW. Cerebroelectrical depression following surfactant treatment in preterm neonates. Pediatrics 1992;89(4 Pt 1):643-7.

\section{Higgins 2008}

Higgins JPT, Green S, editors. Cochrane Handbook for Systematic Reviews of Interventions Version 5.0.1 [updated September 2008]. The Cochrane Collaboration, 2008. Available from www.cochranehandbook.org.

\section{Ho 2002a}

Ho JJ, Subramaniam P, Henderson-Smart DJ. Continuous distending pressure for respiratory distress syndrome in preterm infants. Cochrane Database of Systematic Reviews 2002, Issue 1. [DOI: 10.1002/14651858.CD002271]

\section{Ho 2002 b}

Ho JJ, Henderson-Smart DJ, Davis P. Early versus delayed initiation of continuous distending pressure for respiratory distress syndrome in preterm infants. Cochrane Database of Systematic Reviews 2002, Issue 2. [DOI: 10.1002/14651858.CD002975]

\section{Hughes 1999}

Hughes CA, O'Gorman LA, Shyr Y, Schork MA, Bozynski ME, McCormick MC. Cognitive performance at school age of very low birth weight infants with bronchopulmonary dysplasia. Journal of Developmental and Behavioral Pediatrics 1999;20(1):1-8.

\section{Jobe 1984}

Jobe A, Ikegami M, Jacobs H, Jones S. Surfactant and pulmonary blood flow distribution following treatment of preterm lambs with natural surfactant. Journal of Clinical Investigation 1984;73(3):84856.

\section{Jorch 1997}

Jorch G, Hartl H, Roth B, Kribs A, Gortner L, Schaible T, et al.Surfactant aerosol treatment of respiratory distress syndrome in spontaneously breathing premature infants [letter]. Pediatric Pulmonology 1997;24(3):222-4.

\section{Kamper 1999}

Kamper J. Early nasal continuous positive airway pressure and minimal handling in the treatment of very-low-birth-weight infants. Biology of the Neonate 1999;76(Suppl 1):22-8.

\section{Kilbride 2003}

Kilbride HW, Gelatt MC, Sabath RJ. Pulmonary function and exercise capacity for ELBW survivors in preadolescence: effect of neonatal chronic lung disease. Journal of Pediatrics 2003;143(4): 488-93.

\section{Korhonen 1999}

Korhonen P, Koivisto AM, Ikonen S, Laippala P, Tammela O. Very low birthweight, bronchopulmonary dysplasia and health in early childhood. Acta Paediatrica 1999;88(12):1385-91.

Kribs 2007

Kribs A, Pillekamp F, Hüsneler C, Vierzig A, Roth B. Early administration of surfactant in spontaneous breathing with nCPAP: feasibility and outcome in extremely premature infants (postmenstrual age $\leq 27$ weeks). Paediatric Anaesthesia 2007;17(4): 364-9.

Kribs 2010

Kribs A, Härtel C, Kattner E, Vochem M, Küster H, Möller J, et al.Surfactant without intubation in preterm infants with respiratory distress: first multi-center data. Klinische Pädiatrie 2010;222(1): 13-7.

\section{Kugelman 2007}

Kugelman A, Feferkorn I, Riskin A, Chistyakov I, Kaufman B, Bader D. Nasal intermittent mandatory ventilation versus nasal continuous positive airway pressure for respiratory distress syndrome: a randomized, controlled, prospective study. The Journal of Pediatrics 2007;150(5):521-6.

Lemyre 2002

Lemyre B, Davis PG, De Paoli AG. Nasal intermittent positive pressure ventilation (NIPPV) versus nasal continuous positive airway pressure (NCPAP) for apnea of prematurity. Cochrane Database of Systematic Reviews 2002, Issue 1. [DOI: 10.1002/ 14651858.CD002272]

\section{Majnemer 2000}

Majnemer A, Riley P, Shevell M, Birnbaum R, Greenstone H, Coates AL. Severe bronchopulmonary dysplasia increases risk for later neurological and motor sequelae in preterm survivors. Developmental Medicine and Child Neurology 2000;42(1):53-60.

\section{Marshall 1984}

Marshall T, Deeder R, Pai S, Berkowitz GP, Austin TL. Physiologic changes associated with endotracheal intubation in preterm infants. Critical Care Medicine 1984;12(6):501-3. 
Morley 2008

Morley CJ, Davis PG, Doyle LW, Brion LP, Hascoet JM, Carlin JB, COIN Trial Investigators. Nasal CPAP or intubation at birth for very preterm infants. The New England Journal of Medicine 2008; 358(7):700-8.

\section{O'Reilly 2008}

O’Reilly M, Hooper SB, Allison BJ, Snibson K, Harding R, Sozo F. Airway remodeling and pulmonary inflammation in a model of ventilator-induced injury of the very preterm lung [Abstract]. Journal of Paediatrics and Child Health 2008;44:A63.

\section{Petrikovsky 1995}

Petrikovsky BM, Lysikiewicz A, Markin LB, Slomko Z. In utero surfactant administration to preterm human fetuses using endoscopy. Fetal Diagnosis and Therapy 1995;10(2):127-30.

\section{Pfister 2007}

Pfister RH, Soll RF, Wiswell TE. Protein-containing synthetic surfactant versus animal-derived surfactant extract for the prevention and treatment of respiratory distress syndrome. Cochrane Database of Systematic Reviews 2007, Issue 4. [DOI: 10.1002/14651858.CD006069.pub3]

\section{Reininger 2005}

Reininger A, Khalak R, Kendig JW, Ryan RM, Stevens TP, Reubens $\mathrm{L}$, et al.Surfactant administration by transient intubation in infants 29 to 35 weeks' gestation with respiratory distress syndrome decreases the likelihood of later mechanical ventilation: a randomized controlled trial. Journal of Perinatology 2005;25(11): 703-8.

\section{RevMan 2008}

The Nordic Cochrane Centre, The Cochrane Collaboration. Review Manager 5 (RevMan 5). Copenhagen: The Nordic Cochrane Centre, The Cochrane Collaboration, 2008.

\section{Sarkar 2006}

Sarkar S, Schumacher RE, Baumgart S, Donn SM. Are newborns receiving premedication before elective intubation?. Journal of Perinatology 2006;26(5):286-9.

Seger 2009

Seger N, Soll RF. Animal derived surfactant extract for treatment of respiratory distress syndrome. Cochrane Database of Systematic Reviews 2009, Issue 2. [DOI: 10.1002/14651858.CD007836]

\section{Singer 1997}

Singer L, Yamashita T, Lilien L, Collin M, Baley J. A longitudinal study of developmental outcome of infants with

bronchopulmonary dysplasia and very low birth weight. Pediatrics 1997;100(6):987-93.

Skidmore 1990

Skidmore MD, Rivers A, Hack M. Increased risk of cerebral palsy among very low-birthweight infants with chronic lung disease. Developmental Medicine and Child Neurology 1990;32(4):325-32.

\section{Soll 1998a}

Soll RF. Synthetic surfactant for respiratory distress syndrome in preterm infants. Cochrane Database of Systematic Reviews 1998, Issue 3. [DOI: 10.1002/14651858.CD001149]

\section{Soll 1998b}

Soll RF. Prophylactic synthetic surfactant for preventing morbidity and mortality in preterm infants. Cochrane Database of Systematic Reviews 1998, Issue 2. [DOI: 10.1002/14651858.CD001079]

\section{Soll 1999}

Soll RF. Early versus delayed selective surfactant treatment for neonatal respiratory distress syndrome. Cochrane Database of Systematic Reviews 1999, Issue 4. [DOI: 10.1002/ 14651858.CD001456]

Soll 2001a

Soll RF, Blanco F. Natural surfactant extract versus synthetic surfactant for neonatal respiratory distress syndrome. Cochrane Database of Systematic Reviews 2001, Issue 2. [DOI: 10.1002/ 14651858.CD000144]

\section{Soll 2001b}

Soll RF, Morley CJ. Prophylactic versus selective use of surfactant for preventing morbidity and mortality in preterm infants. Cochrane Database of Systematic Reviews 2001, Issue 2. [DOI: 10.1002/14651858.CD000510]

\section{Soll 2002}

Soll RF. Prophylactic natural surfactant extract for preventing morbidity and mortality in preterm infants. Cochrane Database of Systematic Reviews 2002, Issue 1. [DOI: 10.1002/ 14651858.CD000511]

\section{Soll 2009}

Soll RF, Özek E. Multiple versus single doses of exogenous surfactant for the prevention or treatment of neonatal respiratory distress syndrome. Cochrane Database of Systematic Reviews 2009, Issue 1. [DOI: 10.1002/14651858.CD000141.pub2]

Stevens 2007

Stevens TP, Blennow M, Myers EW, Soll RF. Early surfactant administration with brief ventilation versus selective surfactant and continued mechanical ventilation for preterm infants with or at risk for respiratory distress syndrome. Cochrane Database of Systematic Reviews 2007, Issue 4. [DOI: 10.1002/ 14651858.CD003063.pub3]

Tooley 1987

Tooley WH, Clements JA, Muramatsu K, Brown CL, Schlueter MA. Lung function in prematurely delivered rabbits treated with a synthetic surfactant. American Review of Respiratory Disease 1987; 136(3):651-6.

Trevisanuto 2005

Trevisanuto D, Grazzina N, Ferrarese P, Micaglio M, Verghese C, Zanardo V. Laryngeal mask airway used as a delivery conduit for the administration of surfactant to preterm infants with respiratory distress syndrome. Biology of the Neonate 2005;87(4):217-20.

van de Berg 2009 van de Berg E, Lemmers PMA, Toet MC, Klaessens J, van Bel F. The effect of the "InSurE" procedure on cerebral oxygenation and electrical brain activity of the preterm infant. Archives of Disease in Childhood. Fetal and Neonatal Edition 2010;95(1):F53-8.

\section{Van Marter 2000}

Van Marter LJ, Allred EN, Pagano M, Sanocka U, Parad R, Moore $\mathrm{M}$, et al.Do clinical markers of barotrauma and oxygen toxicity explain interhospital variation in rates of chronic lung disease? The 
Neonatology Committee for the Developmental Network. Pediatrics 2000;105(6):1194-201.

\section{Verder 1994}

Verder H, Robertson B, Greisen G, Ebbesen F, Albertsen P, Lundstrøm K, Jacobsen T. Surfactant therapy and nasal continuous positive airway pressure for newborns with respiratory distress syndrome. Danish-Swedish Multicenter Study Group. The New England Journal of Medicine 1994;331(16):1051-5.

\section{Victorin 1990}

Victorin LH, Deverajan LV, Curstedt T, Robertson B. Surfactant replacement in spontaneously breathing babies with hyaline membrane disease -- a pilot study. Biology of the Neonate 1990;58 (3):121-6.

\section{von der Hardt 2002}

von der Hardt K, Schoof E, Kandler MA, Dotsch J, Rascher W. Aerosolized perfluorocarbon suppresses early pulmonary inflammatory response in a surfactant-depleted piglet model. Pediatric Research 2002;51(2):177-82.

\section{von der Hardt 2004}

von der Hardt K, Kandler MA, Brenn G, et al.Comparison of aerosol therapy with different perfluorocarbons in surfactantdepleted animals. Critical Care Medicine 2004;32(5):1200-6.

\section{Young 2005}

Young KC, Del Moral T, Claure N, Vanbuskirk S, Bancalari E. The association between early tracheal colonization and

bronchopulmonary dysplasia. Journal of Perinatology 2005;25(6): 403-7.

* Indicates the major publication for the study 
CHARACTERISTICS OF STUDIES

Characteristics of excluded studies [ordered by study ID]

\begin{tabular}{ll}
\hline Study & Reason for exclusion \\
\hline Kattwinkel 2002 & Non-randomised study (case series). \\
\hline Kattwinkel 2004 & Non-randomised study (case series). \\
\hline
\end{tabular}


DATA AND ANALYSES

This review has no analyses.

\section{H I S T O R Y}

Protocol first published: Issue 1, 2010

Review first published: Issue 3, 2011

\section{CONTRIBUTIONS OFAUTHORS}

Mohamed E Abdel-Latif and David A Osborn developed the protocol and the review.

DECLARATIONS OF INTEREST

None known

DIFFERENCES BETWEEN PROTOCOL AND REVIEW None. 